\title{
Vibration Control Research for Four Edges Simply Supported Rectangular Thin Plate
}

\author{
Hailin Guo ${ }^{1, \text { a }}$,Shouzhu Hu ${ }^{1, b}$,Guoxing Zhu ${ }^{1, \mathrm{c}}$,Fei Song ${ }^{1, \mathrm{~d}}$, Changli Liu ${ }^{1, \mathrm{e}, \star}$ \\ ${ }^{1}$ School of mechanical and power engineering, East China University of Science and Technology, \\ Shanghai 200237, China;

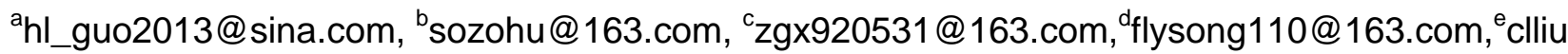 \\ @ecust.edu.cn
}

Corresponding Author: Changli Liu

Keywords:Thin plate,vibration control,kinetic energy of a plate, phase difference.

\begin{abstract}
In this paper, the vibration of power machinery on the deck which is simplified to forced vibration of four edges simply supported rectangular thin plate was studied. The theoretical model of the system is built based on the vibration properties of deck using four-pole parameters method. For analyzing optimal phase relationship among the ship power machines, the deck's kinetic energy is chosen as the objective function. To fulfil active vibration isolation,the phase difference and layout of power machinery on the deck is researched.The analysis result show that this method is simple, reliable and effective to isolate vibration.
\end{abstract}

\section{Introduction}

Vibration control of ships is always the subject studied by researchers [1], however, in the aspect of vibration source research, too much energy is invested in the internal diesel engines and external loads [2] [3] [4], while the machines vibration on deck is ignored. In many cases, the response caused by machinery and devices vibration on deck is continuous, lasting and not avoided, which impact ship's safety, stealthiness and crew's comfort. Especially, intense local vibration would causes fatigue damage to ship structure. Moreover, the ship is developed into more large-scale and complex, which leads to machineries and devices on deck gradually increased, and the problem of ship vibration on deck has become increasingly prominent.

According to F.W.Lanchester patent active vibration absorption principle, vibration absorption devices were applied to the active vibration control of ships in the local vibration. The first vibration absorption device was carried out by W.E.Dal in 1928, while the phase adjustment was manual. Currently, the active vibration control research of a ship deck through active vibration absorption devices worldwide. Dac-seung Cho studied a mechanical vibration isolation device which realized the amplitude adjustment [5], Svensson.Bo. eliminated amplitude and phase adjustable vibration absorption device, which was applied to a real ship test[6]. C.G.Díaz researched the vibration control of a simple thin plate through electromagnetic actuator [7]. In Harbin Engineering University, Han Guangcai, Wang Binqing proposed active mechanical actuator for local vibration control of a ship deck [8] [9].

The method of active vibration reduction is available to conditions of interference and without foreknowledge of vibration source. However, adding actuators will result in extra energy consumption, and the actuators are vibration sources too. In this paper, the ship machine vibration active control research based on the phase modulation method is proposed according to Lanchester's patent principle of active vibration absorption. Without additional actuators, layout and the phase differences of working machines on the deck would be rationally adjusted to achieve damping effect.

\section{Model Building}

In this paper, only the deck's transverse vibration in low frequency is taken into account, and the system is simplified into a simple rectangular thin plate as schematically shown in Fig 1. Assuming 
the weight of $S+1$ machines on the deck is equal to $m$, and one of the forces is regarded as original force $f_{p}=F_{0} e^{j \omega t}$ while the other ones are control forces $f_{c k}=F_{0} e^{j\left(\omega t+\varphi_{c k}\right)},(k=1,2, \mathrm{~L} S)$ where $\varphi_{c k}$ is phase differences between original force and control forces, $\left(x_{p}, y_{p}\right)$ and $\left(x_{c k}, y_{c k}\right)$ are the coordinates of original and control forces.

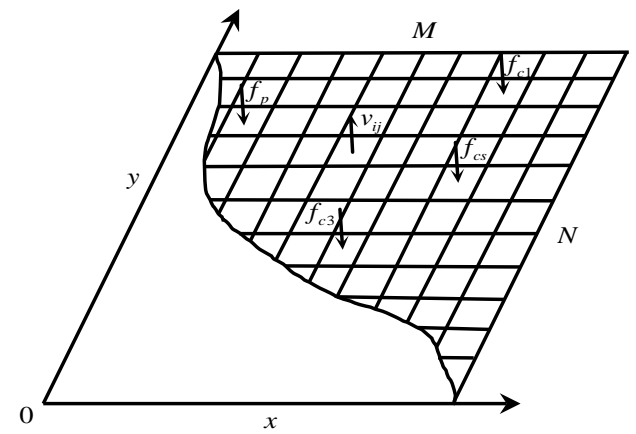

Fig.1 Theoretical Analysis Model

As shown in Fig.1, the mathematical model built in for the simulation considers the plate divided into a grid of rectangular elements whose dimensions have been taken to be $l_{x e}=\frac{a}{M}, l_{y e}=\frac{b}{N}$, where $\mathrm{M}$ and $\mathrm{N}$ are the plate modal orders used in the calculus. The central coordinate of each elements is $\left(x_{e}, y_{e}\right)$, where $x_{e}=\frac{(2 i-1) a}{2 M}, y_{e}=\frac{(2 j-1) b}{2 N}(i=1,2, \mathrm{~L}, M, j=1,2, \mathrm{~L}, N)$. The phasors of the control force $F_{c}$ is:

$$
F_{c}=\left[\begin{array}{llll}
f_{c 1} & f_{c 2} & \mathrm{~L} & f_{c S}
\end{array}\right]^{T}
$$

The vibration of the plate at the centers of the elements can be expressed in matrix form using mobility functions:

$$
v_{i, j}=Y_{p, e} f_{p}+Y_{c, e} F_{c}
$$

Where the components of the velocity/force mobility matrices, $Y_{p, e}, Y_{c, e}$, between the position of forces and elements, are given by:

$$
Y_{p(c), e}=\frac{4 j \omega}{\rho h a b} \sum_{m=1}^{\infty} \sum_{n=1}^{\infty} \frac{\sin \left(\frac{m \pi x_{p(c)}}{a}\right) \sin \left(\frac{n \pi y_{p(c)}}{b}\right) \sin \left(\frac{m \pi x_{e}}{a}\right) \sin \left(\frac{n \pi y_{e}}{b}\right)}{\omega_{m n}^{2}(1+j \delta)-\omega^{2}}
$$

Where $\rho, h, a, b$ is density, thickness, length and width of the plate. $j=\sqrt{-1} . \delta$ is the hysteresis loss factor. $m, n$ is the modal indices. $\omega_{m n}=\pi^{2}\left(\frac{m^{2}}{a^{2}}+\frac{n^{2}}{b^{2}}\right) \sqrt{\frac{D}{\bar{m}}}$ is the natural frequency of the $(m, n)$ th bending mode where $\bar{m}=\rho h$ is surface density of elements, $D=\frac{E h^{3}}{12\left(1-\mu^{2}\right)}$ is stiffness of the plate where $E$ is Modulus of Elasticity and $\mu$ is Poisson ratio.

This expression can be approximated to the summation of the kinetic energies of each element into which the plate has been subdivided so that

$$
\begin{gathered}
E_{i, j}(\omega)=\frac{M_{e}}{4} v_{i, j}^{H} v_{i, j} \\
E(\omega)=\sum_{i=1}^{M} \sum_{j=1}^{N} E_{i, j}(\omega)
\end{gathered}
$$

Where $M_{e}=\rho h l_{x e} l_{y e}$ is the mass of the elements and $H$ denotes the Hermitian transpose.

\section{Numerical Analysis}

Geometry and physical parameters of the plate is shown in Table 1. Two exciters have been taken into account respectively to investigate the phase relation and layout of the two excitation have influence on the total kinetic energy of the plate. 
Table 1 Geometry and physical parameters of the plate

\begin{tabular}{llll}
\hline Parameter & Symbol & Value & Unit \\
\hline Dimension & $a \times b$ & $800 \times 500$ & $\mathrm{~mm}$ \\
Thickness & $h$ & 3 & $\mathrm{~mm}$ \\
Density & $\rho$ & $7.8 \times 10^{3}$ & $\mathrm{Kg} / \mathrm{m}^{3}$ \\
Young's modulus & $E$ & $2.1 \times 10^{11}$ & $\mathrm{~N} / \mathrm{m}^{2}$ \\
Poisson's ratio & $\mu$ & 0.25 & - \\
Damping loss factor & $\delta$ & 0.02 & - \\
Member of elements & $M \times N$ & $40 \times 25$ & - \\
\hline
\end{tabular}

\subsection{Impact of control force position.}

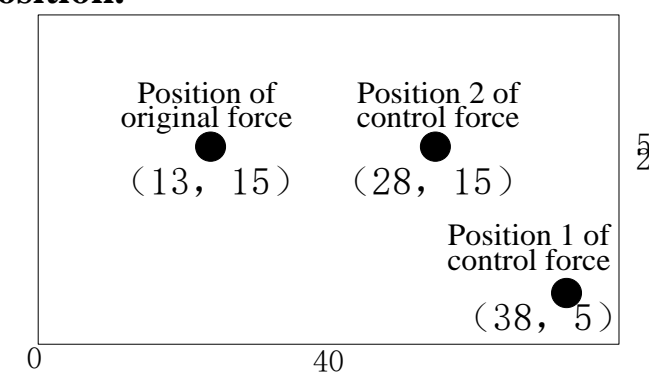

Fig.2Layout of forces

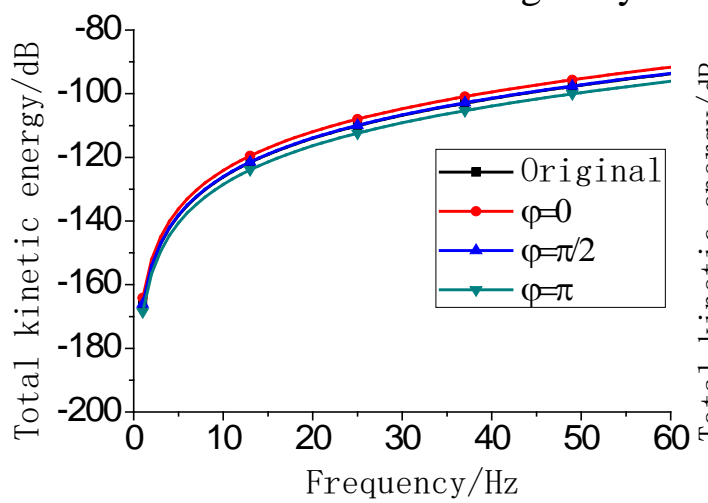

(b)Position 1

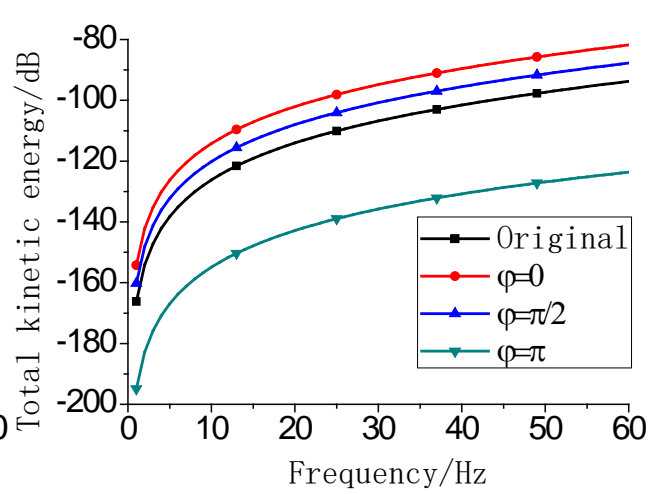

(a) Position 2

Fig.3 Amplitude-frequency figure of the total kinetic energy of the plate

As is showed inFig. $2, f_{p}$ is fixed on position $(13,15)$ and $f_{c 1}$ is respectively fixed on position $(38,5)$ and $(28,15)$.Amplitude-frequency figure of the total kinetic energy of the plate is showed in Fig.3. Fig.3(a) shows that the phase difference has little impact on the total kinetic energy of the plate when $f_{c 1}$ is fixed on position $(38,5)$, while Fig.3(b) shows that the total kinetic energy of the plate has declined $25 \mathrm{~dB}$ when $\varphi_{1}=\pi$.

Fig. 3 shows that the total kinetic energy of the plate is influenced by the position of the control force and the phase difference between control force and original force, and the influence extent of phase difference is related to the coordinate of control force. In summary, the vibration control of the plate based on the phase modulation method was restricted that the control force should be placed on appropriate position. The influencing factor, the position of the control force, has been researched as following.

When $\omega=50 \mathrm{~Hz}$ and $f_{p}$ is fixed on $(5,10),(30,15)$, according to the change of the position of $f_{c 1}$, the total kinetic energy of the plate under different phase difference is shown inFig. 4. Fig. 4shows that the total kinetic energy of the plate approach to the optimal value on a annular region which includes the original force point when $\varphi 1=\pi$.According to the fitting results, the optimal path of the control force is an ellipse that through the original force point, whose center is on point (20.52, 12.99). Therefore, the optimal path of the control force can be described as

$$
\frac{\left(x_{c}-20.52\right)^{2}}{x_{p}^{2}-41 x_{p}+2.6 y_{p}^{2}-67.34 y_{p}+858.5}+\frac{\left(y_{c}-12.99\right)^{2}}{0.386 x_{p}^{2}-15.83 x_{p}+y_{p}^{2}-25.98 y_{p}+331.2}=1
$$




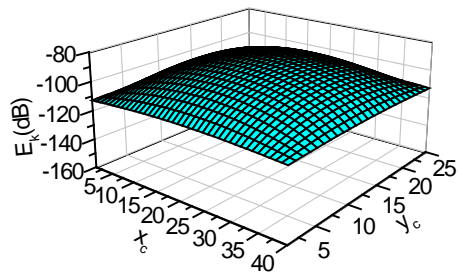

(a) 0

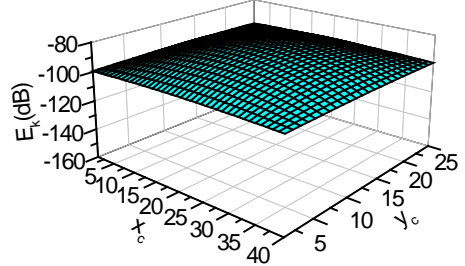

(d) 0

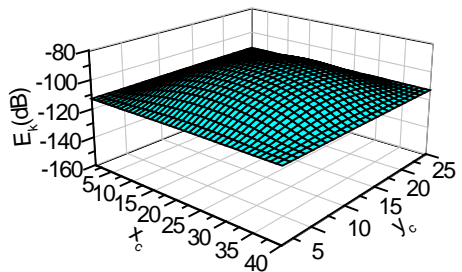

(b) $\pi / 2$

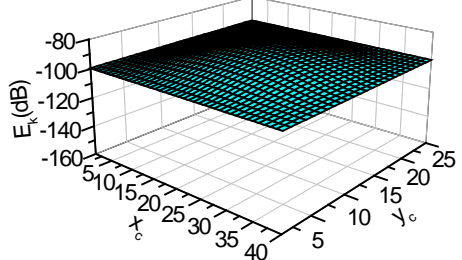

(e) $\pi / 2$

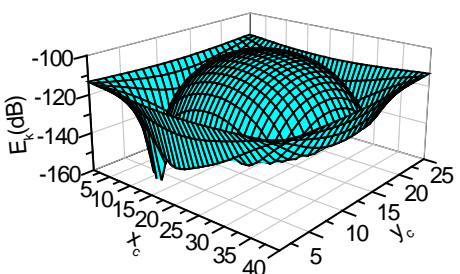

(c) $\pi$

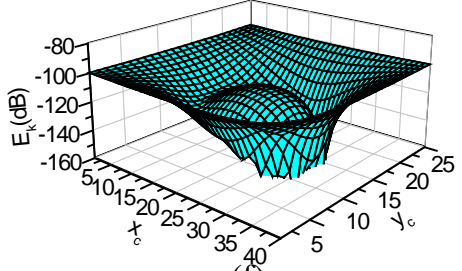

(f) $\pi$

(a), (b), (c) denote the position of original force is fixed on $(5,10)$ and (d),(e),(f) denote the position of original force is fixed on $(5,10)$.

\subsection{Impact of phase difference.}

Fig.4Distribution of the total kinetic energy of the plate

The Fig. 5shows the total kinetic energy of the plate excited by original force and control force in case of different phase when $\omega=50 \mathrm{~Hz}$ and the position of original force is $\mathrm{O}(15,10)$ and the position of control force is separately fixed on points $\mathrm{A}(14,11), \mathrm{B}(18,17)$ and $\mathrm{C}(27,11)$ at random according to optimal control path described above.

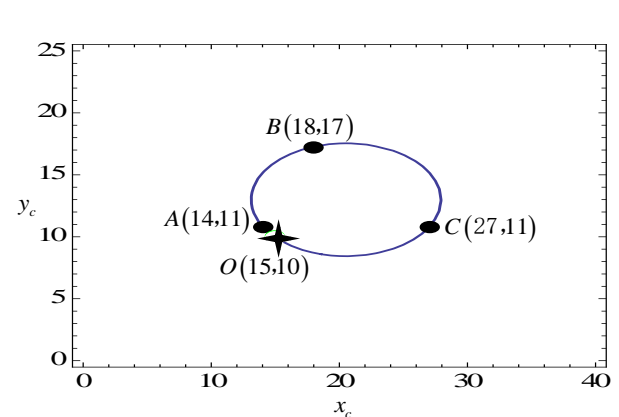

(a) positions of forces

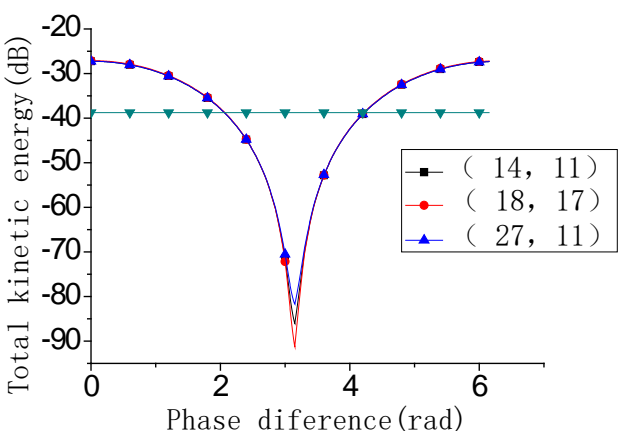

(b) vibration of the plate

Fig. 5 Impact of phase difference

As is showed inFig. 5, the critical phase difference is $2 \pi / 3$. In other words, when phase difference is $2 \pi / 3$,the total kinetic energy of the thin plate excited by two forces is equal to which is excited only by original force. It could be concluded that the vibration of the plate would be effectively controlled when phase difference varies from $2 \pi / 3$ to $4 \pi / 3$, and the total kinetic of the plate decline rapidly and reach the minimum value when $\varphi=\pi$.

\section{Summary}

In this paper, theoretical and experimental analysis are presented to research the active vibration control of the deck based on phase-adjustment method. Firstly, the system is simplified to forced vibration of a four-edge simply supported plate that excited by several concentrated harmonic load randomly distributed. Then, the total kinetic energy of the plate is calculated as the objective function to investigate the vibration of the plate. Finally, two factors, the layout and the phase difference of the load that affect the total kinetic energy of the plate have been taken into consideration.The result shows that it is not effective to control the vibration of the plate through phase-adjustment method only when the load are positioned on a specific elliptical path, and it will access to the best damping effect when $\varphi=\pi$. 


\section{References}

[1] LIWei-jia, CAO Qing-song. Advances and Review on the Research ofthe Active Control of Ship Vibration[J]. SHIPBUILDING 0F CHINA, 2007,02:68-79.

[2] ZHANG Hongtian,LIU Zhigang,WANG Zhiqiu.An active eletro-dynamic vibration absorber equipment and its application to diesel vibration control[J],Journal of Heilongjiang Institute of Technology, 2003,01:3-8.

[3] YANG Tiejun,LI Xinhui,ZHU Minggang. Experimental investigation of actiVe Vibration control for diesel engine generators in marine applications[J]. Journal of Vibration Engineering, 2013,02:160-168.

[4] Mitsuhashi K, Biwa T, Mizuhara S. Application of active vibration isolating system to diesel engine mounting[A]. 18th International Congress on combustion Engines, Diesel Engines Volume[C].Tianjing, China, 1989: 281300.

[5] Dae-Seung Cho,Soo-Mok Lee,Kyoon-Yang Chung. Ship vibration control using a force-adjustable mechanical actuator. Journal of Vibration and Control,1999,5(5):779-794P.

[6] Svensson.Bo.Compensators Designed to Reduce Vibration.Diesel and Gas Turbine Publications, 2003: 14-35P.

[7] Cristóbal González Díaz, Christoph Paulitsch, Paolo Gardonio.Active damping control unit using a small scale proof mass electrodynamic actuator[J]. Journal of the Acoustical Society of America,2008,124(2):886-897.

[8] HAN Guangcai.The applied study of mechanical actuator on vibration control of ship[D]. Harbin Engineering University,2005.

[9] WANG Binqing.The Study of active vibration control technique based on mechanical actuator[D].Harbin Engineering University,2005. 